\title{
Strongly interacting matter under intense magnetic fields.
}

\section{Pablo G. Allen}

Department of Theoretical Physics, GIyA, CNEA, Libertador 8250, (1429) Buenos Aires,

Argentina.

E-mail: pabloallen1dgmail.com

\section{Norberto N. Scoccola*}

Department of Theoretical Physics, GIyA, CNEA, Libertador 8250, (1429) Buenos Aires,

Argentina.

Universidad Favaloro, Solís 453, (1078) Buenos Aires, Argentina.

CONICET, Rivadavia 1917, (1033) Buenos Aires, Argentina.

E-mail: Scoccoladtandar.cnea.gov.ar

We study the effects of magnetic fields on the phase diagram of strongly interacting matter using the Polyakov-Nambu-Jona-Lasinio model. We consider two parameter sets showing that they lead to rather different results, especially at low temperatures.

X Latin American Symposium on Nuclear Physics and Applications (X LASNPA),

1-6 December 2013

Montevideo, Uruguay

\footnotetext{
*Speaker.
} 


\section{Introduction}

Understanding the behavior of strongly interacting matter under the influence of intense magnetic fields has become an issue of increasing interest in recent years [W]. This is mostly motivated by the realization that in some relevant physical situations, like high energy non-central heavy ion collisions [[] and compact stellar objects called magnetars [3], very strong magnetic fields may be produced. Since in these systems extreme temperatures and/or densities may be found, it is interesting to investigate which modifications are induced by the presence of strong magnetic fields on the whole QCD phase diagram. Unfortunately, even in the absence of those fields, the present knowledge of such phase diagram is only schematic due to the well-known difficulty given by the so-called sign problem which affects lattice calculations at finite chemical potential[可]. Of course, the presence of strong magnetic fields makes the situation even more complex. Thus, most of our present knowledge of their effect comes from investigations performed in the framework of effective models (see e.g. [5] and refs. therein). In this contribution we present some results of a study in the framework of a two-flavor Polyakov-Nambu-Jona-Lasinio (PNJL) model[6]. This type of model is constructed by incorporating the Polyakov Loop (PL) into the finite temperature and chemical potential Nambu-Jona-Lasinio (NJL) model. The NJL model[ $[\square]$ is an effective relativistic quark model for non perturbative QCD, where gluon degrees of freedom are integrated out and interactions are modelled through point like scalar and pseudo scalar interactions that describe chiral symmetry breaking effectively. On the other hand, the PL dynamics is used to represent the phenomena of confinement.

\section{Formalism}

The Euclidean PNJL action coupled to the EM field reads

$$
S_{E}=\int d^{4} x\left\{\bar{\psi}\left(-i \gamma^{\mu} D_{\mu}+m_{0}\right) \psi-G\left[(\bar{\psi} \psi)^{2}+\left(\bar{\psi} i \tau \gamma_{5} \psi\right)^{2}\right]+\mathscr{U}(\Phi[\mathscr{G}(x)])\right\}
$$

where $m_{0}$ is the current mass and $G$ is a coupling constant. Since the model under consideration is not renormalizable, we need to specify a regularization scheme. Here, we introduce a sharp cut-off, $\Lambda$, in 3-momentum space, only for the divergent ultra-violet integrals. The set of values for $m_{0}, G$ and $\Lambda$ completely determine the quark sector of the model. In this publication we consider two sets of parameters. The corresponding parameter values for Set 1 (Set 2) are $m_{c}=5.595$ (5.833) $\mathrm{MeV}, \Lambda=620.9$ (587.9) $\mathrm{MeV}$ and $G \Lambda^{2}=2.212(2.440)$. The coupling of the quarks to the (electro)magnetic field $\mathscr{A}$ and the gluon field $\mathscr{G}$ is implemented via the covariant derivative $D_{\mu}=$ $\partial_{\mu}-i q_{f} \mathscr{A}_{\mu}-i \mathscr{G}_{\mu}$ where $q_{f}$ represents the quark electric charge $\left(q_{d}=-q_{u} / 2=-e / 3\right)$. We consider a static and constant magnetic field in the $z$ direction, $\mathscr{A}_{\mu}=\delta_{\mu 2} x_{1} B$. Concerning the gluon fields, we assume that quarks move on a constant background field $\phi=\mathscr{G}_{4}$. Then the traced Polyakov loop, which in the infinite quark mass limit can be taken as an order parameter of confinement, is given by $\Phi=\frac{1}{3} \operatorname{Tr} \exp (i \phi / T)$. We work in the so-called Polyakov gauge, in which the matrix $\phi$ is given a diagonal representation $\phi=\phi_{3} \lambda_{3}+\phi_{8} \lambda_{8}$. This leaves only two independent variables, $\phi_{3}$ and $\phi_{8}$. In the case of vanishing chemical potential, owing to the charge conjugation properties of the QCD lagrangian, the traced Polyakov loop in the Mean Field Approximation (MFA) is expected to be a real quantity. Since $\phi_{3}$ and $\phi_{8}$ have to be real valued, this condition implies $\phi_{8}=0$. In order 
to have real values for the MFA thermodynamical potential for finite $\mu$ we set $\phi_{8}$ also in that case. The mean field traced Polyakov loop reads then $\Phi=\Phi^{*}=\left[1+2 \cos \left(\phi_{3} / T\right)\right] / 3$. To proceed we need to specify the explicit form of the Polyakov loop effective potential $\mathscr{U}(\Phi, T)$. Here we consider the form based on the logarithmic expression of the Haar measure associated with the SU(3) color group integration. It reads [[]]

$$
\mathscr{U}(\Phi, T)=-(1 / 2) a(T) \Phi \Phi^{*}+b(T) \ln \left(1-6 \Phi \Phi^{*}+4\left(\Phi^{3}+\left(\Phi^{*}\right)^{3}\right)-3\left(\Phi \Phi^{*}\right)^{2}\right)
$$

where

$$
a(T)=a_{0}+a_{1}\left(T_{0} / T\right)+a_{2}\left(T_{0} / T\right)^{2}, \quad b(T)=b_{3}\left(T_{0} / T\right)^{3} .
$$

The values of the constants $a_{i}, b_{3}$ can be fitted to pure gauge lattice QCD results. One obtains $a_{0}=3.51, a_{1}=-2.47, a_{2}=15.2, b_{3}=-1.75$. The dimensionful parameter $T_{0}$ in Eq.(R.3]) corresponds in principle to the deconfinement transition temperature in the pure Yang-Mills theory, $T_{0}=270 \mathrm{MeV}$. However, it has been argued that in the presence of light dynamical quarks this temperature scale should be adequately reduced [Q]. Correspondingly, we will consider the value $T_{0}=208 \mathrm{Mev}$ in our calculations.

Finally, using the Matsubara formalism to account for finite temperature and chemical potential in the quark sector, the MFA thermodynamical potential for the models under consideration reads

$$
\Omega=\Omega_{V A C}+\Omega_{M A G}+\Omega_{M E D}+\mathscr{U}(\Phi, T)
$$

where

$$
\begin{aligned}
\Omega_{V A C} & =\frac{\left(M-m_{0}\right)^{2}}{4 G}-\frac{N_{c} N_{f}}{\pi^{2}} \int_{0}^{\Lambda} d p p^{2} \sqrt{p^{2}+M^{2}} \\
\Omega_{M A G} & =-\frac{N_{c}}{2 \pi^{2}} \sum_{f=u, d}\left(q_{f} B\right)^{2}\left[\zeta^{\prime}\left(-1, x_{f}\right)+\frac{x_{f}^{2}}{4}-\frac{1}{2}\left(x_{f}^{2}-x_{f}\right) \log x_{f}\right] \\
\Omega_{M E D} & =-\frac{T}{2 \pi} \sum_{s, k, c, f} \alpha_{k}\left|q_{f}\right| B \int_{-\infty}^{+\infty} \frac{d p_{z}}{2 \pi} \ln \left[1+\exp \left(-\frac{E_{f}\left(p_{z}, k\right)+s \mu+i \phi_{c}}{T}\right)\right] .
\end{aligned}
$$

Here, $M$ is the dressed quark mass. In addition, $s= \pm 1, \alpha_{k}=2-\delta_{k 0}, x_{f}=M^{2} /\left(2\left|q_{f}\right| B\right), E_{f}\left(p_{z}, k\right)=$ $\sqrt{M^{2}+p_{z}^{2}+2 k\left|q_{f}\right| B}$, and $\phi_{c}=\phi_{3}, \phi_{3},-2 \phi_{3}$ for $c=r, g, b$, respectively.

\section{Cold quark matter under strong magnetic fields}

We consider first the case of finite chemical potential and vanishing temperatures. In this situation, $\Phi=0$ and, thus, the PL decouples leaving only the dressed quark mass $M$ to be determined for given values of $\mu$ and $B$. This is done by solving the gap equation, $\partial \Omega / \partial M=0$. As discussed below, the behavior of these solutions as a function of $\mu$ indicates the existence of some kind of phase transition. In the chiral case (i.e. $m_{c}=0$ ) they can be of first or second order depending on the value of the external magnetic field. For finite current masses, however, the possible second order phase transitions become smooth crossovers. Consequently, there is not a unique way to define their position. In fact, even in the absence of a magnetic field, different prescriptions have been used in the literature to define the position of a crossover-type transition. Here, we will use the location of the peak of the chiral susceptibility $\chi_{c h}=\partial<\bar{q} q>/ \partial m_{c}$. 
Some typical results of the behavior of $M$ as a function of the chemical potential for different values of $e B$ are shown in Fig. $\mathrm{W}$. It is always found that at a low enough value of $\mu$, the system
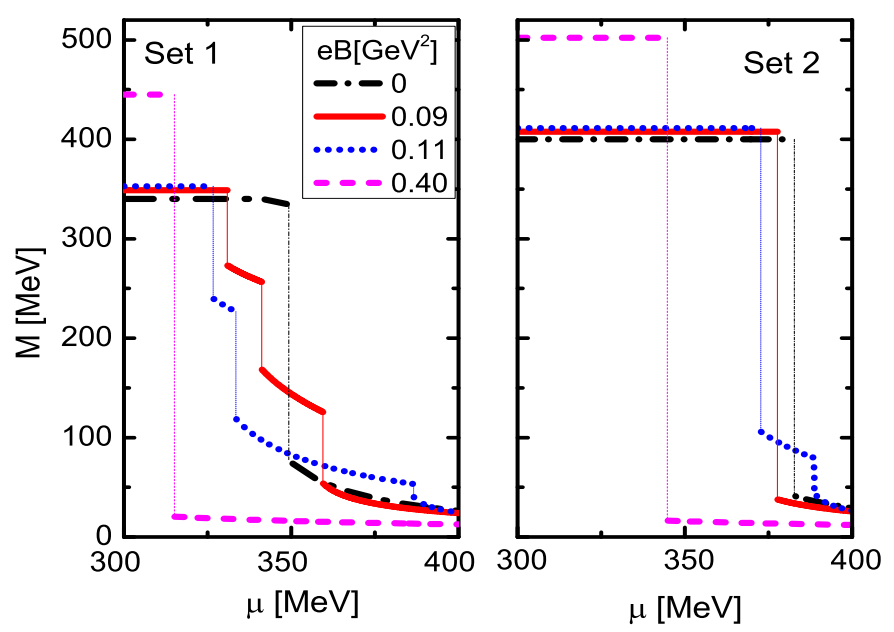

Figure 1: $M$ vs $\mu$ for several $e B$ values for Set 1 and Set 2 .

will be in a broken symmetry vacuum phase, where the quark mass is large and independent of the chemical potential. If we increase the chemical potential, we find a series of transitions whose nature, number and magnitude basically depend on the chosen parameter set and magnetic field value [ए]]. It is generally seen that the presence of a magnetic field induces a rich phase structure, which in turn becomes simpler for high $M_{0}$ values. Here, $M_{0}$ is the value of the dressed quark mass for $\mu=T=B=0$. For example, for $B=0$ in Set $2\left(M_{0}=400 \mathrm{MeV}\right)$, the chirally restored phase is achieved through a single transition, while for finite values of magnetic field, we see that it is possible to encounter more than one phase transition before reaching the fully restored symmetry phase. For Set $1\left(M_{0}=340 \mathrm{MeV}\right)$, the situation is far more complex. On the one hand, there are two transitions for the $B=0$ case: first, at $\mu=340 \mathrm{MeV}$, there is a second order transition where mass starts to decrease slightly and density also becomes finite. At a slightly larger value of $\mu$, there is a discontinuous drop to the restored symmetry phase. For finite values of $B$, we see that the restored phase is reached via multiple transitions, for not very large values of the magnetic field. This complex behavior is concisely represented in Fig. \, where the phase diagrams in the $\mu-e B$ plane have been plotted for the two sets of parameters under consideration. Full (black) lines represent first order transitions, while red (dashed) lines represent crossover transitions. The $\mathrm{B}$ - phase is the broken symmetry phase were the quark density is zero. In both $\mathrm{A}_{i}$ and $\mathrm{C}_{i}$ phases, Landau levels are populated, being chiral symmetry partially restored in $\mathrm{C}_{i}$ and fully restored in the $\mathrm{A}_{i}$ phases. In the non vacuum region, every phase transition produces the population of some new quark level: if $i$ is an even number, there is a non-zero population for levels up to $i$ for $d$-quarks and $i / 2$ for $u$-quarks, while if $i$ is an odd number, population is non zero up to $i$ for $d$-quarks and $(i-1) / 2$ for $u$-quarks.

Our results indicate the existence of certain critical value $M_{0}^{\text {crit }} \simeq 360 \mathrm{MeV}$ [ए0]. For parametrizations leading to $M_{0}>M_{0}^{\text {crit }}$ we could say that there is one unique transition that separates the fully 

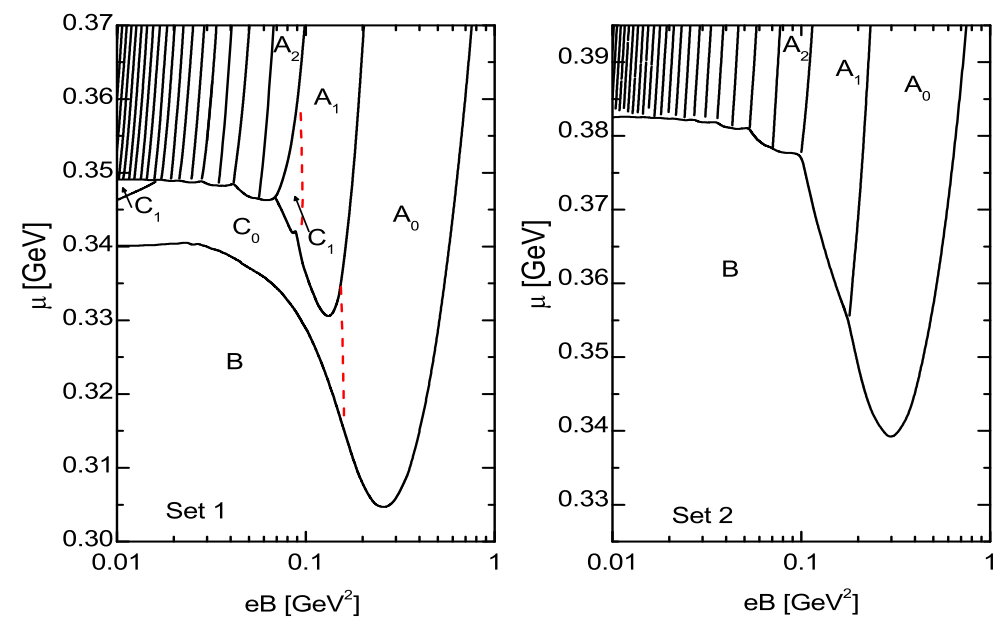

Figure 2: Phase Diagrams in the $\mu-e B$ plane for Set 1 and Set 2. Full (black) lines indicate first order transitions, and dashed (red) lines indicate crossover transitions.

broken chiral symmetry phase $\mathrm{B}$ from the $\mathrm{A}_{i}$ phases, where symmetry is restored. On the other hand, for parametrizations leading to $M_{0}<M_{0}^{\text {crit }}$, as chemical potential is increased several phase transitions are typically found before symmetry is fully restored.

\section{Magnetized quark matter at finite temperature and chemical potential}

At finite $T$ the dressed quark mass and the PL are obtained as solutions of the coupled gap equations $\partial \Omega / \partial M=\partial \Omega / \partial \Phi=0$. Moreover, in the case of crossover transitions, the deconfinement pseudo-critical temperature is defined by the location of the peak of the PL susceptibility $\chi_{\Phi}=d \Phi / d T$.

In Fig. [1], the phase diagrams in the $\mu-T$ plane for several values of the magnetic field are plotted, for both parameter sets. There is a main chiral phase transition (black line) which is typically divided into two parts: at lower chemical potential values it is of crossover type (dashed line), while it is first order (full line) for higher values of $\mu$. The crossover line and the first order line meet at a critical end point. The deconfinement phase transition is only considered in the low-intermediate chemical potential region, i.e. in the region where the chiral transition is of the crossover type. In this range of values, it is generally seen that the deconfinement transition occurs at a lower temperature than the chiral transition, and that, while the former is relatively independent of the magnetic field, the latter moves towards higher temperatures as magnetic field is increased. The latter behavior, although common to the one found in most effective models, disagrees with the recent LQCD findings[ $[\mathbb{]}]$. The intermediate/high temperature range is qualitatively similar for both parameter sets, but for low temperature there is a phase structure that is highly dependent on the behavior at zero temperature. As seen in Sec. 3, for Set 1 the phase structure at zero temperature is quite more complicated at intermediate magnetic fields, with more phase transitions separating the broken symmetry phase from the fully restored symmetry phase. It is seen that these transi- 

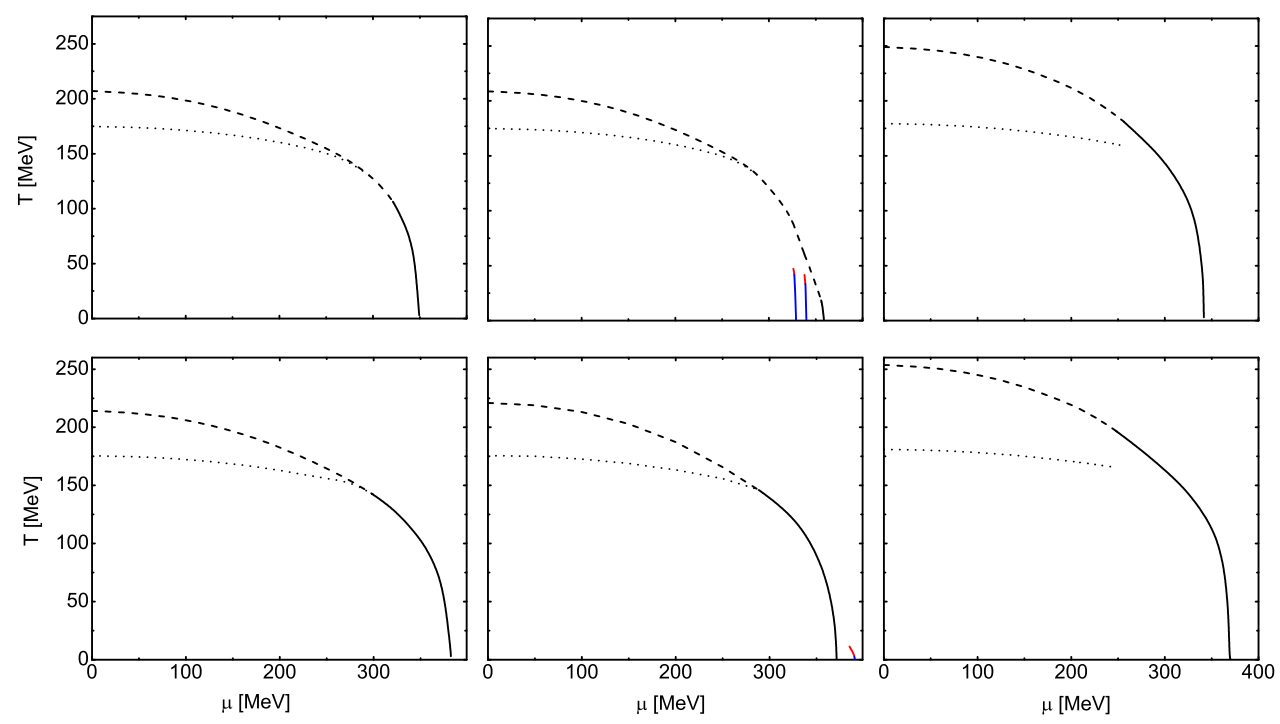

Figure 3: Phase diagrams in the $T-\mu$ plane for Set 1 and Set 2 and several magnetic fields. Upper panels: Set $1 B=0$ (left), $e B=0.089 \mathrm{GeV}^{2}$ (center), $e B=0.6 \mathrm{GeV}^{2}$ (right). Lower panels: Set $2 B=0$ (left), $e B=0.11 \mathrm{GeV}^{2}$ (center), $e B=0.6 \mathrm{GeV}^{2}$ (right). Full (black) lines indicate first order phase transitions, dashed lines indicate chiral crossover transitions, dotted lines indicate deconfinement crossover transitions, full red lines indicate secondary first order chiral transitions and full blue lines indicate secondary crossover chiral transitions.

tions continue to exist as first order in a certain range of temperatures above zero (full blue lines), resulting again in a series of consecutive transitions connecting different phases as the chemical potential is increased. These transitions have a critical end point after which they become crossover type transitions (full red lines). These ones in turn are washed away after a small further increase in the temperature. In Set 2, there is not such a complicated structure, however, after chiral symmetry has been restored, the first order transitions amongst $A_{i}$ phases occurring at zero temperature are observed to still exist for very small temperatures, once again becoming crossovers at a CEP. In this diagram, the first of these transitions is seen. All of these multiple transitions above discussed are related to the presence of Landau Levels, hence there is none of this behavior for zero magnetic field.

\section{Conclusions}

In this work we have presented results for the behavior of quark matter under intense magnetic fields in the framework of the PNJL model. In the cold quark matter case, when studying the behavior of the order parameter as a function of $\mu$, the $B=0$ case presents only a few possible phases. For a low enough chemical potential, symmetry is fully broken and for high chemical potential it is restored through a first order phase transition. For finite $B$, these two phases still exist, but a new set of phases may exist at intermediate chemical potential where symmetry is only partially restored and successive transitions occur as $\mu$ increases so as to populate Landau Levels. Depending on the magnetic field and parameter set (characterized in this work by $M_{0}$, i.e. the value 
of the dressed quark mass for $\mu=T=B=0$ ), one may encounter a different number of these transitions. It is generally seen that the phase diagram is simplified when changing the parameter set so that $M_{0}$ adjusts to a higher value. In the finite temperature case, both chiral and deconfinement transition were also studied. As already known, chiral transitions in the $\mu-T$ plane are of crossover type in the lower $\mu$ range and become first order transitions for higher $\mu$. These two transitions meet at a critical end point. In this model, the deconfinement transition is relatively independent of the magnetic field, and occurs always at lower temperatures than the chiral transition. The latter moves towards higher temperatures as magnetic field is increased in agreement with the results of most effective models. However, recent LQCD calculations [U] have shown quite the opposite effect, namely that the $T_{c h}$ actually decreases with temperature. It is believed [ए]2] that the reaction of the gluon sector to the presence of an external magnetic field should be incorporated in effective models in order to describe this so-called "inverse magnetic catalysis". In fact, it has been argued [ए]3] that, to some extent, this can be done by using a PL potential which depends on the magnetic field in the framework of an enhanced model where further couplings are allowed between Polyakov and quark fields. For high temperatures, the phase diagram is relatively independent of the parameter set, but for low temperatures, the phase structure seen at $T=0$ reflects on the finite temperature region, giving rise to several first order transitions, each with a corresponding critical end point that leads to a crossover.

This work has been partially funded by CONICET (Argentina) under grants PIP 00682 and by ANPCyT (Argentina) under grant PICT-2011-0113.

\section{References}

[1] D.E. Kharzeev, K. Landsteiner, A. Schmitt and H.-U. Yee, Lect. Notes Phys. 871, 1 (2013).

[2] D.E. Kharzeev, L.D. McLerran and H.J. Warringa, Nucl. Phys. A803, 227 (2008).

[3] R.C. Duncan and C. Thompson, Astrophys J. 392, L9 (1992).

[4] F. Karsch, E. Laermann, Quark Gluon Plasma 3, edited by R.C.Hwa et al. (World Scientific Singapore), p.1., (2004).

[5] E.S. Fraga, Lect. Notes Phys. 871, 121 (2013); R. Gatto and M. Ruggieri, Lect. Notes Phys. 871, 87(2013).

[6] K. Fukushima, Phys. Lett. B591, 277 (2004); C. Ratti, M.A. Thaler and W. Weise, Phys. Rev. D73, 014019 (2006); E. Megias, E. Ruiz Arriola and L.L. Salcedo, Phys. Rev. D74, 065005 (2006).

[7] U. Vogl and W. Weise, Prog. Part. Nucl. Phys. 27, 195 (1991); S. Klevansky, Rev. Mod. Phys. 64, 649 (1992); T. Hatsuda and T. Kunihiro, Phys. Rep. 247, 221 (1994).

[8] S. Roessner, C. Ratti, and W. Weise, Phys. Rev. D75, 034007 (2007).

[9] B.-J. Schaefer, J.M. Pawlowski and J. Wambach, Phys. Rev. D76, 074023 (2007).

[10] P.G. Allen and N.N. Scoccola, Phys. Rev. D88, 094005 (2013).

[11] G.S.Bali et al, J. High Energy Phys. 02044 (2012); G.S.Bali et al, Phys. Rev. D86, 071502 (2012).

[12] F. Bruckmann, G. Endrodi and T.G. Kovacs, J. High Energy Phys. 1304, 112 (2013).

[13] M. Ferreira, P. Costa, D.P. Menezes, C. Providencia, N.N . Scoccola, Phys. Rev. D89, 016002 (2014). 